\title{
Development of a GSM-Based Environmental Monitoring System
}

\author{
${ }^{1}$ Oke A.O., ${ }^{+2}$ Awokola J.A., ${ }^{3}$ Amusan E.A. \\ ${ }^{1,+2,3}$ Department of Computer Science and Engineering. \\ Ladoke Akintola University of Technology, Ogbomoso, Oyo State, Nigeria
}

\begin{abstract}
This paper proposes the design and application of temperature sensor to free the stress of continuous check on the environment for any change in temperature. This can help in having an accurate reading of changes in temperature over a particular period of time. The instability in temperature of an environment (for example server rooms of data centers) can cause damage, and also reduce the efficiency and life span of equipments found in the data centers. An alert sub-system and a Global system for mobile communication (GSM) module was incorporated into the design to make it more effective. This environmental monitoring system was designed to monitor the temperature at certain range (either increase or decrease) and it is powered by an external source of power supply via an AC to DC power adapter.
\end{abstract}

Keywords: GSM, Sensor, Microcontroller, LCD

\section{INTRODUCTION}

In recent years, there has been a growing concern about technology all over the world on how it has provided man with important solutions as well as created other problems for man. In lieu of these problems, man has been responsible and acquainted with exposing themselves to the much needed experience in the handling of equipment and machines with respect to their various fields. However, further exposure to other technologies has helped humanity in battling these problems.

One of such technologies that has helped in environmental monitoring are Sensors (both wired and wireless types). Wireless sensors and sensor networks are now being considered as substitute for traditional tethered monitoring systems. The benefit of wireless monitoring systems is that they are inexpensive to install because extensive wiring is not required between sensors and the data acquisition system. Today, wireless sensors offer impressive computational resources for processing data. Hardware only represents one half of the complete wireless sensing unit design and software embedded in the wireless sensors represent the second half.

However, wireless monitoring systems like temperature sensors, water sensors and the likes have proved to be inefficient due to unstable supply of electricity that feeds them especially in the context of developing nations. Some researchers are exploring the development of power free wireless sensors known as Radio- Frequency identification (RFID) sensors. RFID sensors are passive radio energy emanated from a remote reader so that it can communicate its measurement back with computational power coupled with the sensors. These wireless sensors are capable of autonomous operation.

A sensor is an essential device that responds to some environmental variable and converts it into electrical output. This signal may then need to be conditioned (filtered, amplified, attenuated, converted) to allow the Microcontroller unit (MCU) to receive the input in a usable form. Digital sensors may provide a direct input at Transistor-transistor Logic (TTL) levels, while some analogue inputs might need a high-performance amplifier or complex digital processing.

Tseng et al in 2006 described a feasibility study on application of GSM-SMS technology to field data acquisition. This paper represents a study on GSM-SMS technology application to the data acquisition. This is a prototype system which is composed of field monitoring and host control platforms. Data transmission and communication are performed by GSM-SMS methodology. The paper indicates the format of short message which is suitable for monitoring the area and field data collection such as (temperature, humidity etc.). The paper revolves around the new development in wireless sensor technologies that is suitable for wireless communication as applied to wireless sensors in the monitoring of agriculture and food production. The work underscored the advantages of 
wireless sensor and reasons for their fast adoption. Wireless sensors are suitable for applications, such as monitoring dangerous/hazardous remote areas and locations. This technology provides nearly unlimited installation flexibility for sensors and increased network robustness.

Cao et al. in 2006 analyzed Remote Meter-Reading System which is based on Wireless Communication Technology. This work represents GSM and Bluetooth technology. The meter reading systems as well as its hardware and software components were described in details. Bluetooth technology defined information that can be get from meters and sensor control. GSM network communicated between intelligent terminal and management center.

Li et al. in 2011 designed a wireless sensor network that is based on water-level monitoring system and its implementation. Water-level monitoring has been widely used to reduce the danger of disasters and ensure the safety of ship channels as well as monitor and control the aquatic environment. This work defines that remote water level monitoring system (RWMS). The RWMS has real-time remote monitoring. RWMS consist of sensor module, data centre module, base station module and web releasing module. It has excellent features in the form of advantages like anti-jamming and expand abilities capabilities. This system gave excellent result in Poyanghu Lake.

Palafox et al in 2011 gave a solution of SMS based water level monitoring system .This work is helpful as a warning device in flood water level monitoring during typhoons and in heavy downpours. At critical water levels, it can send message to the associated device which will then sound an alarm. The sensors are made up of wires which are connected to make a simple switch. When the sensor is triggered, it follows the instructions and the GSM modem will send warning messages at each level.

Anies et al in 2009 provided a detailed tank water level monitoring system by using GSM Network. The paper presents the development of water level monitoring system with an integration of GSM module to alert the person through Short Message Service (SMS). Ramani et al in 2002 defined and give solution of water level for bore well by using GSM. In earlier times, there was no method for monitoring water level. Farmers had to face very difficult situations and they work for the whole day to switch the pump motor off but they cannot sort the solution. It can solve by GSM and it will automatically give alarm sound to mobile user. In this paper it describes its solution when the water level in the bore well drops threshold below or rises to the threshold level for pumping.

\section{BACKGROUND STUDIES}

Pandikumar et al in 2013 proposed a novel architecture based on GSMSMS. This architecture has two major parts that is measuring unit and base station. Here the GSM wireless technology is used to transfer data in a secured channel from measuring unit to base station. The measurement unit has gas, temperature and humidity sensors, GPS receiver and GSM modem. The prototype model has four input channel and ADC. The ADC has $4 \times 1$ multiplexer that converts sensors analog value into digital data. The microcontroller reads GHG parameters and location coordination then constructs green SMS that it will send to base station. Their paper presented an embedded system design of wireless sensor monitoring system for sensing and computation of environmental parameters. A Co2 commercial sensor had been integrated with ARM processor to monitor and compute the level of existence of GHG parameters (like Co2, temperature and humidity) in atmosphere using information and communication technologies. Prototype operations for data gathering and data transmission using GSM-SMS and preliminary test prove that the developed prototype is capable to monitor and compute Co2, temperature and humidity parameters in the deployed environment and has several advantages in term of fast delivery, zero data loss, low cost, flexibility, user friendliness and energy efficiency. The established GSM network is highly efficient and the average SMS delivery time is $3.5 \mathrm{sec}$. This greenhouse monitoring system will create awareness, perform scientific studies and forecast remediation policies by the authorities to individuals and organization in controlling global warming and GHG parameters.

Usama Abdullah and Ayesha Ali (2014) used sensors to provide monitoring solution in the form a system that can monitor water level and sensing temperature via SMS by using GSM technology. This system has capability to detect water level of the tank and sense the temperature of the tank. It notifies GSM modem to send SMS to intended user phone. The PIC microcontroller used as central processor which is connected to the modem by using MAX 232 interface to check the microcontroller operation. A series of tests are organized and the system was found to be working properly. However, delay in receiving SMS can occur due to detecting the circuit and the programming of PIC. 


\section{DESIGN}

This environmental monitoring system was designed to monitor the temperature at certain range (either increase or decrease) and it is powered by an external source of power supply via an AC to DC power adapter. The power adapter converts the alternating current of $220 \mathrm{~V} / 240 \mathrm{~V}$ into a direct current of $5 \mathrm{~V}$ as required to power the device. The System consists of the Hardware and software. The Hardware is divided into six sections: Power supply, Temperature sensor, Microcontroller, Liquid crystal display (LCD), Buzzer, GSM module while the software program was written in C language. The code was written in such a way that it embeds the High and Low sensitivity of the sensor, the buzzing unit, Light emitting diode (LED), LCD and the programmed GSM module. The block diagram of an environmental monitoring system is as shown in the figure1 below:

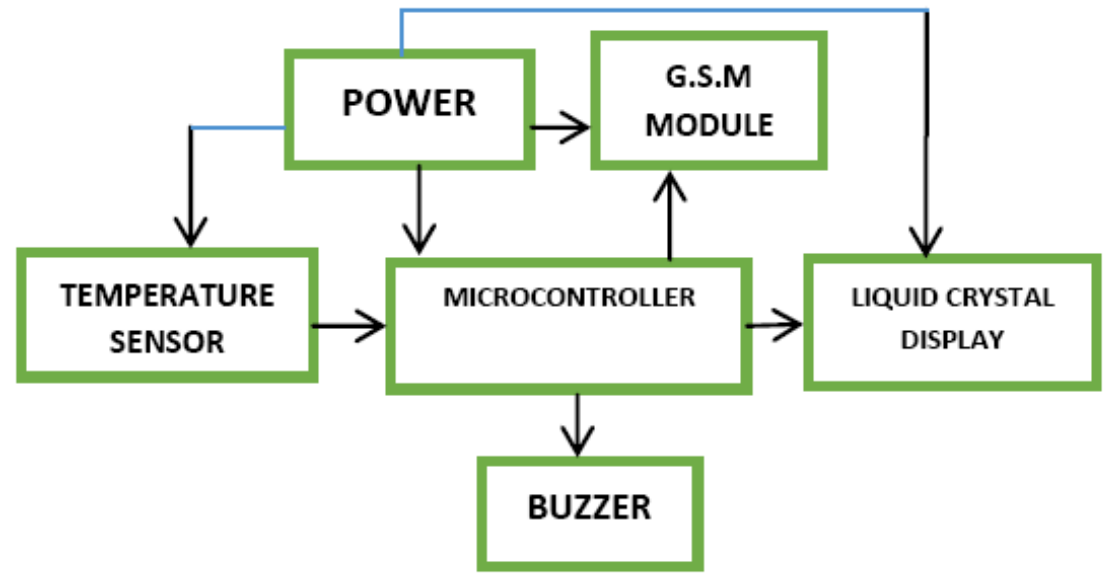

Fig1. Block diagram of an environmental monitoring system.

\section{Temperature Sensor}

As the name implies, this unit is responsible for sensing and interpreting the value of the measured parameter. The temperature sensor used is Thermistor which serve as Transducer that helps convert heat energy in temperature form to electrical energy. The active component that makes up the temperature sensor is LM35 $\left(0^{\circ} \mathrm{c}\right.$ to $\left.100^{\circ} \mathrm{c}\right)$ temperature monitoring Integrated circuit as shown in Fig 2. It is a calibrated integrated circuit temperature sensor operating at two terminals Zener. The IC has a broken voltage directly proportional to absolute temperature at $+110 \mathrm{mV} /{ }^{0} \mathrm{~K}$. With than $1 \Omega$ dynamic impedance, the device operates over a current range of $400 \mu \mathrm{A}$ to $5 \mathrm{~mA}$ with virtually no change in performance when calibrated at $25^{\circ} \mathrm{C}$. The $\mathrm{LM} 35$ has a typical less than $1{ }^{\circ} \mathrm{C}$ error over $100^{\circ} \mathrm{C}$ range on like other sensor; the LM35 has a linear output. This property make interfacing to a readout or control circuitry especially more easy. It works with the principle of thermistors.

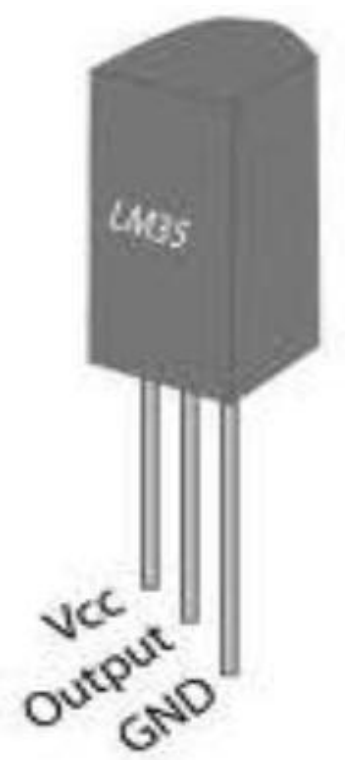

Fig2. Temperature sensor (LM35) 
Thermistors are made from a single piece of semiconductor material, where the charge carrier has mobility; therefore the resistance depends on temperature. The response is exponential, giving a relatively large change for a small change in temperature, and a particularly high sensitivity. Unfortunately, it is non-linear, so is difficult to convert for precise measurement purposes. The thermistor therefore tends to be used as a safety sensor to detect if a component such as a motor or transformer is overheating. The bead type could be used with a comparator to provide warning of overheating in a microcontroller output load.

There are two Types of Thermistors:

- Negative Temperature Coefficient (NTC) Thermistor.

- Positive Temperature Coefficient (PTC) Thermistor.

The NTC thermistors are the thermistors whose resistance decreases proportionally with increase in temperature. They are commonly made from oxides of metals such as manganese.

The PTC thermistors are thermistors whose resistance increases with temperature. They are used in cases where a certain range of temperature is not to be exceeded. In this work, a PTC thermistor was used. The thermistor (LM35) used is under a contact temperature sensor. It was preferred because it has a high sensitivity to small temperature changes. This change will be noticed by the PIC which contains an analogue to digital converter that converts it to its equivalent digital value.

\section{$>$ Microcontroller}

The microcontroller is simply a computer on a chip. It is one of the most important developments in electronics since the invention of the microprocessor itself. It is essential for the operation of devices such as mobile phones, DVD players, video cameras, and most self-contained electronic systems. The small LCD screen is a good clue to the presence of a Microcontroller Unit (MCU). It needs a programmed device to control it. It works sometimes with other chips, but often on its own. The MCU provides the key element in the vast range of small programmed devices which are now commonplace.

However, the PIC16F877 has a good range of features and allows most of the essential techniques. It has a set of serial ports built in, which are used to transfer data to and from other devices, as well as analogue inputs which allow measurement of inputs such as temperature. All standard types of microcontrollers work in a similar way, so analysis of one will make it possible to understand all the others. The PIC16F877 is also a good choice for learning about micro-controllers because the programming language is relatively simple compared to a microprocessor such as the Intel Pentium which is used in the PC. This has a powerful but complex instruction set to support advanced multimedia applications.

The supporting documentation for the PIC MCU is well designed. The mobile phone can support a microcontroller application in addition to the sophisticated digital communications subsystem which provides its main function. It can also have a full-color, medium resolution liquid crystal display (LCD) screen, camera, sound system and so on. However, an Arduino board was used, which is also a microcontroller board on the ATmega328. This can be powered via the USB connection or with an external power supply with an operating voltage of 5v. It has 14 digital input/output pins. Fig.3shows PIC16F877A.

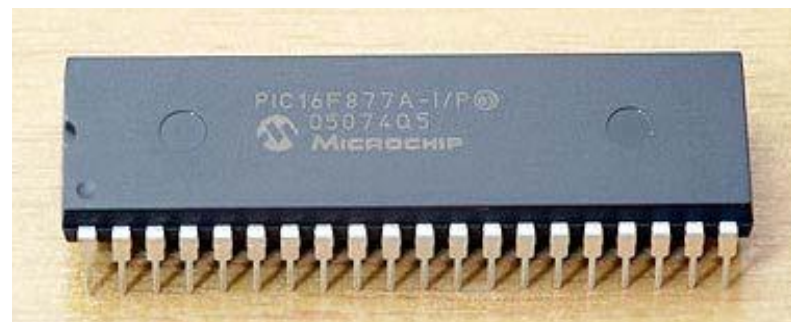

Fig3. PIC16F877A Microcontroller

\section{$>$ Liquid Crystal Display}

Liquid Crystal Display (LCD) is a flat panel display, electronic visual display or video display that uses the light modulating properties of liquid crystals. Liquid crystals do not emit light directly. LCDs 
are used in a wide range of applications including computer monitors, televisions, instrument panels, aircraft cockpit displays, and signage. They are common in consumer devices such as video players, gaming devices, clock, watches, calculators and telephones.

The operation of the 8 character LCD is connected in 4-bit mode that is ASCII codes are fed to it in two stages (high nibble then low nibble). Thus, the data inputs are connected to the high bits of Port D. The low bits provide the control lines RS (Register Select) and E (Enable). The RW (Read/Write) line is connected low for writing only. It is not necessary to use the LCD handshaking, which would require a change in data direction and make the software more complex.

Moreover, the LCD operates as follows: a control byte is presented at the data inputs and RS set low to select command mode. A pulse on $\mathrm{E}$ then latches the high nibble, with the low nibble following in the same way. The command mode is used for operations such as resetting the cursor position to the first character, or clearing the display. The ASCII character codes are loaded by taking RS high for data mode, and latching the code in two stages. The display can be initialized to auto-increment the cursor to the next space on the same line when a character is added to the line, but needs a specific command to go to the second line. Fig. 4 shows a liquid crystal display.

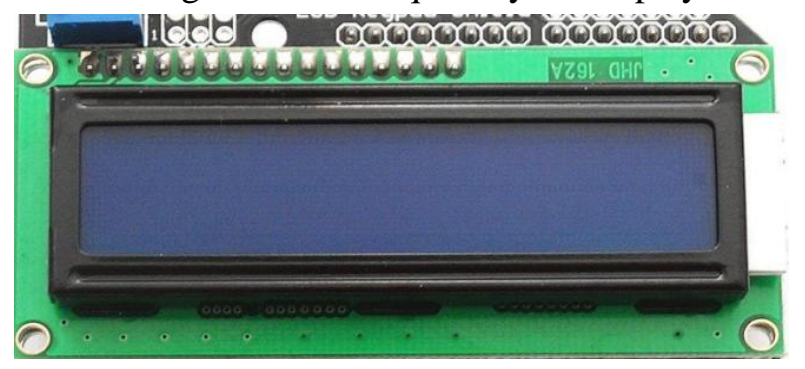

Fig4. The LCD used

\section{$>$ GSM Module}

A Sony Ericsson K700i cell phone was connected to work as GSM modem, which supports AT commands and a serial connection with cable. In the PIC 16F877, the USART is accessed through pins RB6 and RB7. It has two modes of operation, synchronous (using a separate clock signal) and asynchronous (no clock connection). The asynchronous mode is probably used more often, as other methods of synchronous transmission are available in the PIC. In asynchronous mode, RB6 acts as a data transmit (TX) output and RB7 as data receive input (RX). Data is usually transmitted in 8-bit words with the least significant bit sent first. A Sony Ericsson K700i was used in transmitting and Tecno M7 was used in Receiving. Sony Ericsson K700i was used for transmitting because its modem is easy to work with than any other phone. At the Receiving end, any phone can be used to receive. Fig 5 shows the GSM module used in transmitting.

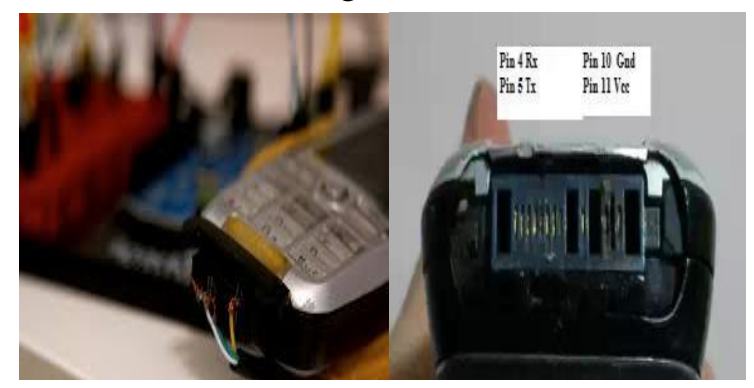

Fig5. The GSM module

\section{$>$ The Software}

The environmental monitoring system was implemented using $C$ language (version 1.05). The code was generated and debugged in C language 2013 environment. Arduino 1.0.5 software was used in writing the codes. The code was written in such a way that it embeds the High and Low sensitivity of the sensor, the buzzing unit, LED, LCD and the programmed GSM module. The main program which consists of the whole programming is located in control unit of the microcontroller and saved. The reason for choosing $\mathrm{C}$ over other languages is its speed, stability and near universal availability. There is an option to program and re-program the IC (PIC16F877) a thousand times to perform different functions. Fig. 6 shows the $C$ language Interface; Fig. 7 shows when the code was run. Fig. 8 shows the Circuit diagram of An EMS. 
Oke A.O. et al.

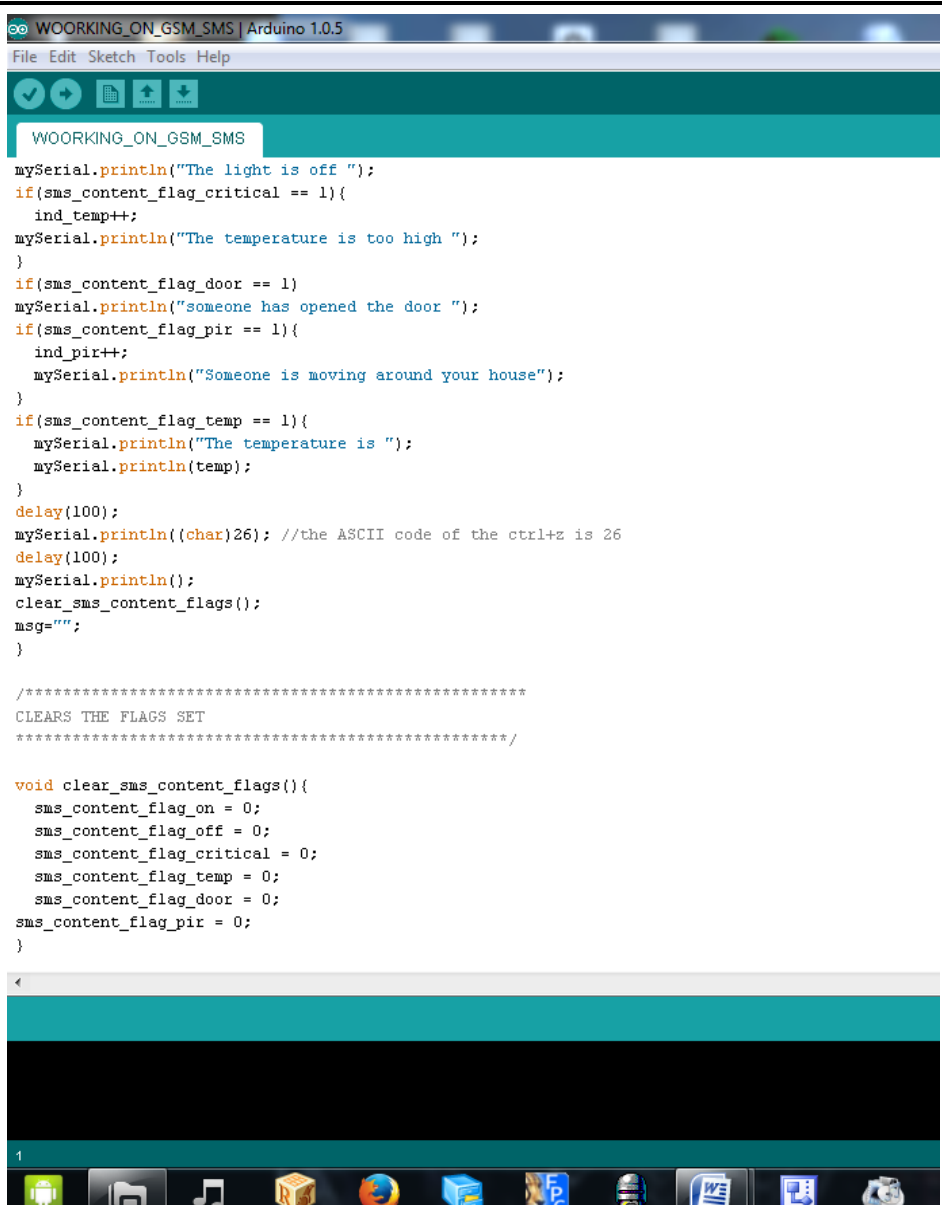

Fig6. C language Interface

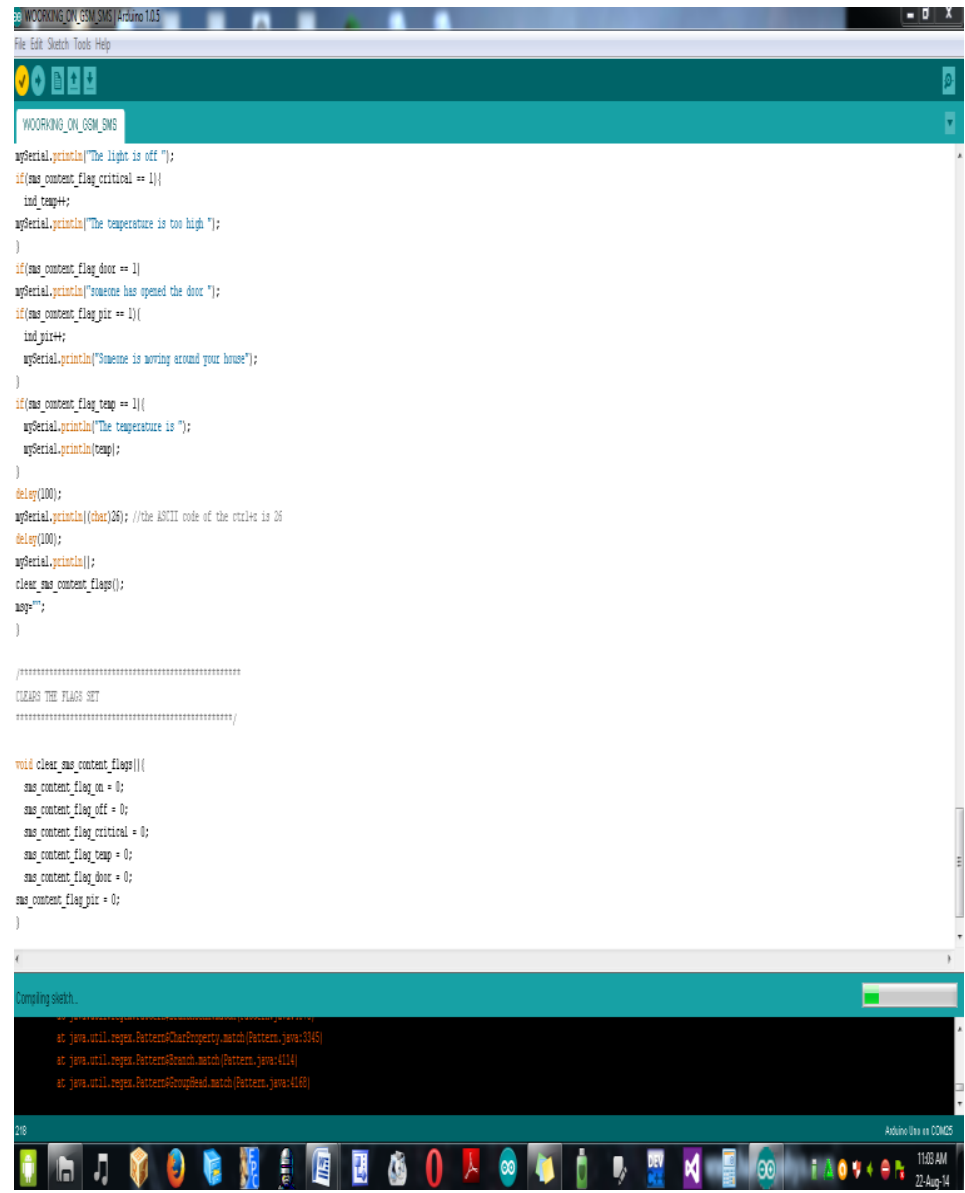

Fig7. Running of the code 


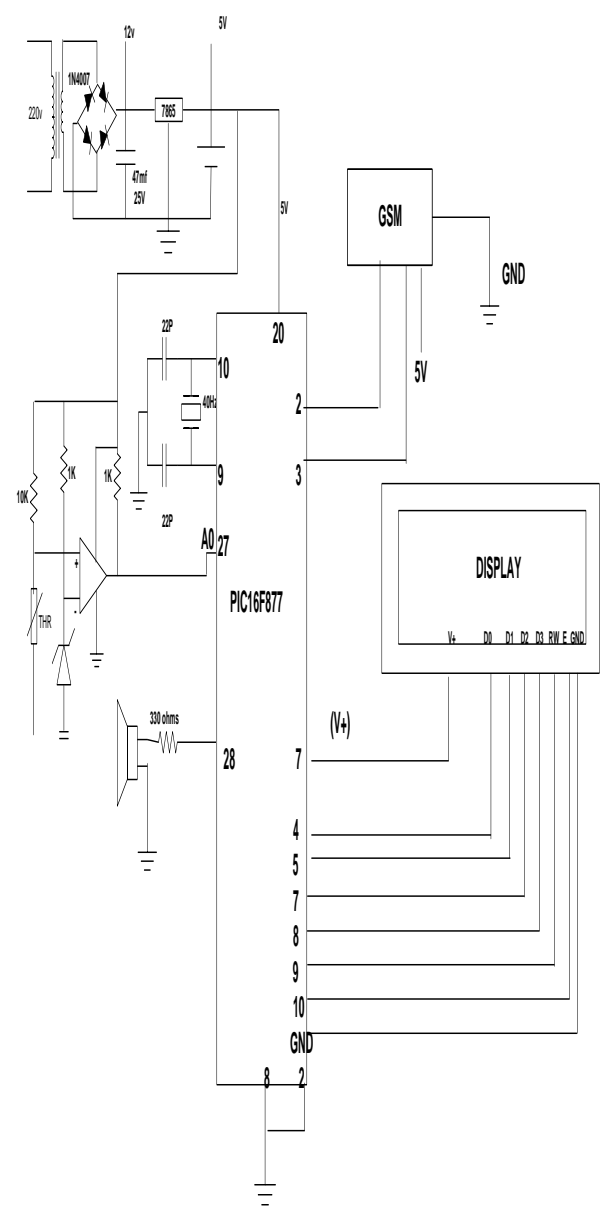

Fig8. The circuit diagram of EMS

\section{CONSTRuCTION AND Discussion}

\section{Construction}

This is the aspect of the work that deals with assembling, soldering, casing etc. The circuit components used was locally sourced. Once they were gotten, the components were then assembled and soldered on a Ferro board according to the specifications of the circuit diagram.

After soldering, the testing of the circuit design took place across the nodes of the circuit. A side of the casing had to be drilled in order for the power jack to fit in and for ventilation. After drilling, the parts were positioned in such a way that they were exposed to the external environment and they were held solidly to the case with the use of an adhesive.

\section{Operation of the System}

The designed device signals when a specific temperature $\left(-10^{0} \leq \Theta \geq 30^{\circ}\right)$ has been exceeded from that of the room temperature and reports by sending a message from the device to the programmed GSM number, buzz from the buzzer and display from the LCD while providing warnings at programmed temperature settings. The detailed operation of the system is stated in the following steps as follows;

\section{STEP1}

The environmental monitoring device operates in such a way that when the power is supplied to the designed system, the sensor senses the temperature of the environment in an analogue form. The programmed microcontroller converts the analogue input to a digital form to be used by the control unit for necessary data flow. The liquid crystal display (LCD) helps in displaying the measured temperature in degree centigrade. The red spot represent the Light emitting diode (LED) which notify when temperature is exceeded, while the black spot represent the sensor used. Fig. 9 shows power not supplied to EMS and Fig. 10 shows the Display on LCD when Power was supplied to EMS. 
Oke A.O. et al.

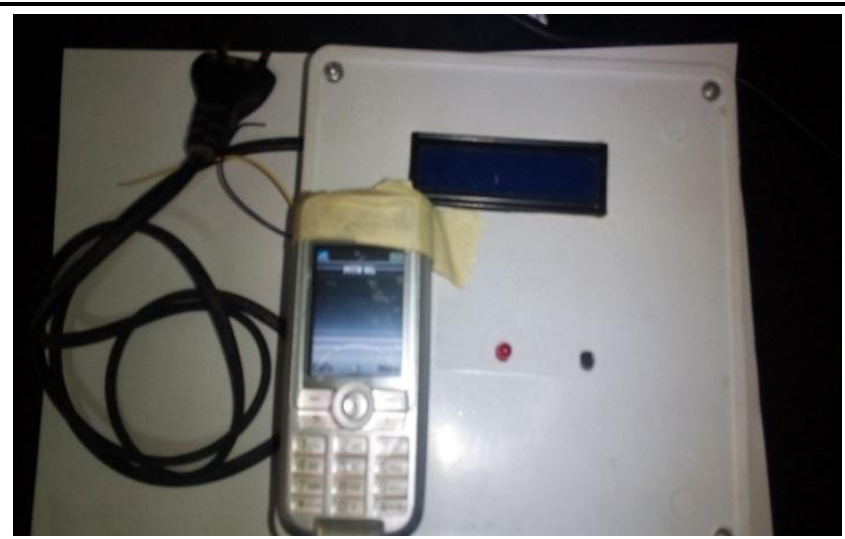

Fig9. Power not supplied to EMS

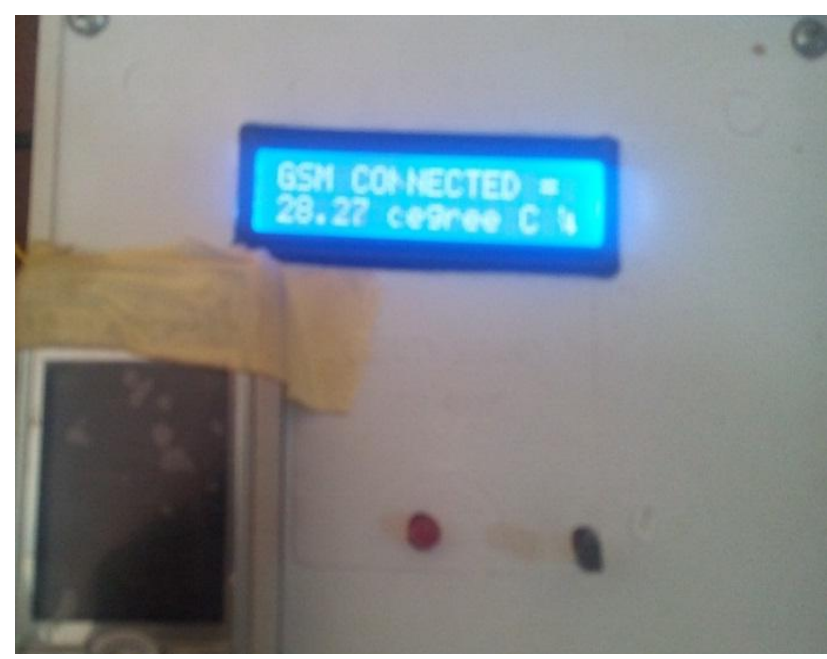

Fig10. Power Supplied to EMS

\section{STEP2}

A case where the temperature range that has been programmed in the microcontroller (for low temperature $\leq-10^{\circ} \mathrm{C}$ and for high temperature $\geq 30^{\circ} \mathrm{C}$ ) was exceeded is observed as follows. A soldering iron was used as a tester during the implementation of the EMS. A soldering iron is a hand tool used in soldering. It supplies heat which is needed in testing the functions of the EMS. The reading on the LCD display is $34.99^{\circ} \mathrm{c}$ which is above the specific range of $30^{\circ} \mathrm{c}$ for this work. The LED notifies with the light. Fig. 11 shows when the environmental temperature has been exceeded.

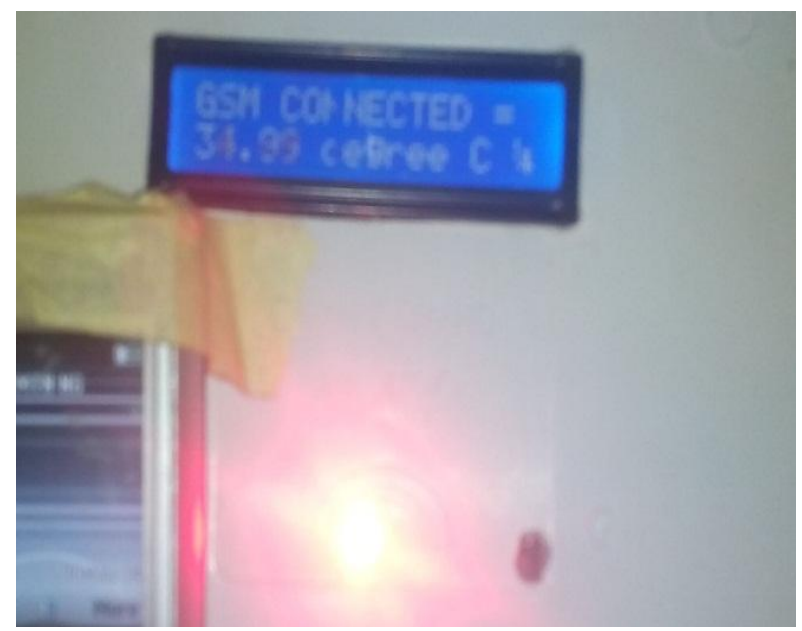

Fig11. Temperature exceeded

\section{STEP3}

This shows the LED signals when the Temperature has been exceeded. The readings show $32.90^{\circ} \mathrm{c}$. Fig.12 shows the LED 


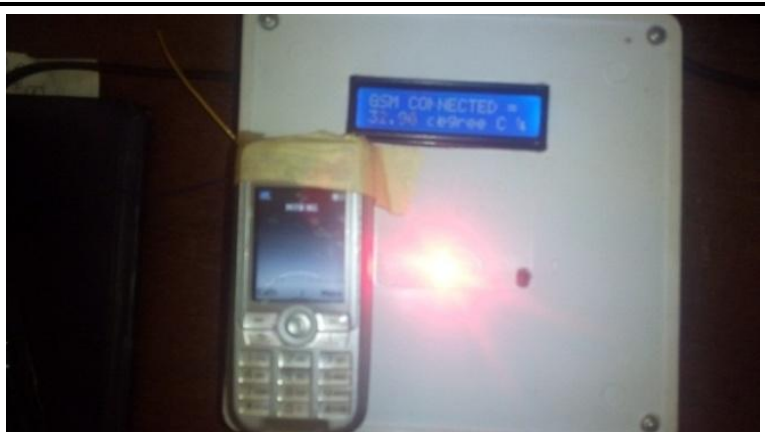

Fig12. LED Signals

\section{STEP4}

For this given ranges the buzzer gives an audio signal or sound output to alert the personnel in charge. Fig 13 shows the message sent to the receiving phone "Temperature Exceeded"

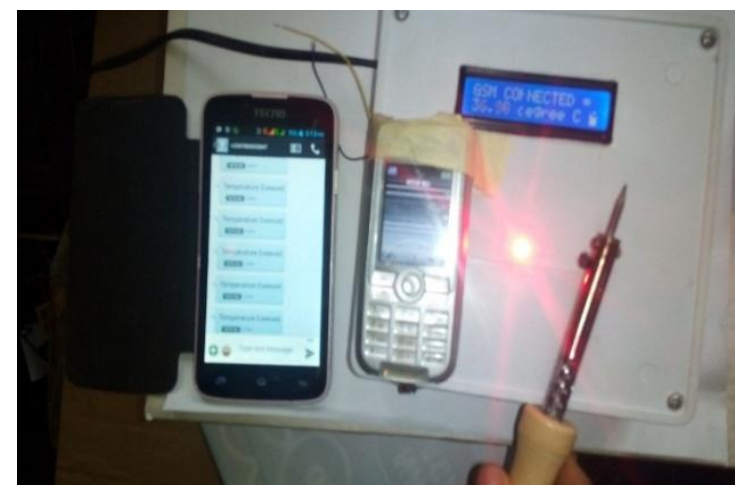

Fig13. EMS Buzz and send SMS

\section{STEP5}

The microcontroller interacts with the modem of the GSM module when the temperature range is exceeded and sends SMS (Temperature Exceeded) to the programmed phone number. Fig. 14 shows the message displayed on the receiving phone

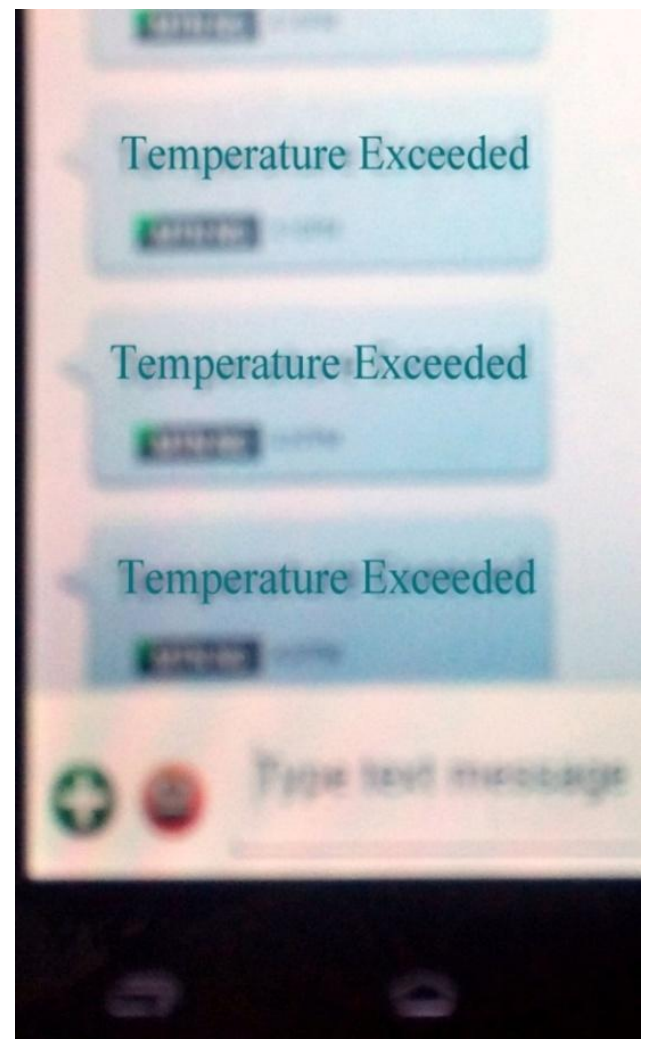

Fig14. SMS received on phone 
Oke A.O. et al.

At the end of the experiment, the designed system has been tested at different time of the day (morning, afternoon and night) and the system was found to be capable of measuring the changes in temperature of the environment. Plate 1 shows the temperature readings in the morning, Plate 2 shows the temperature readings in the afternoon and Plate 3 shows the temperature readings at night. The temperature measured by the device is illustrated below:

Temperature measured (morning) $=21.45^{\circ} \mathrm{c}$

Temperature measured $($ afternoon $)=28.38^{\circ} \mathrm{c}$

Temperature measured $($ night $)=23.06^{\circ} \mathrm{c}$

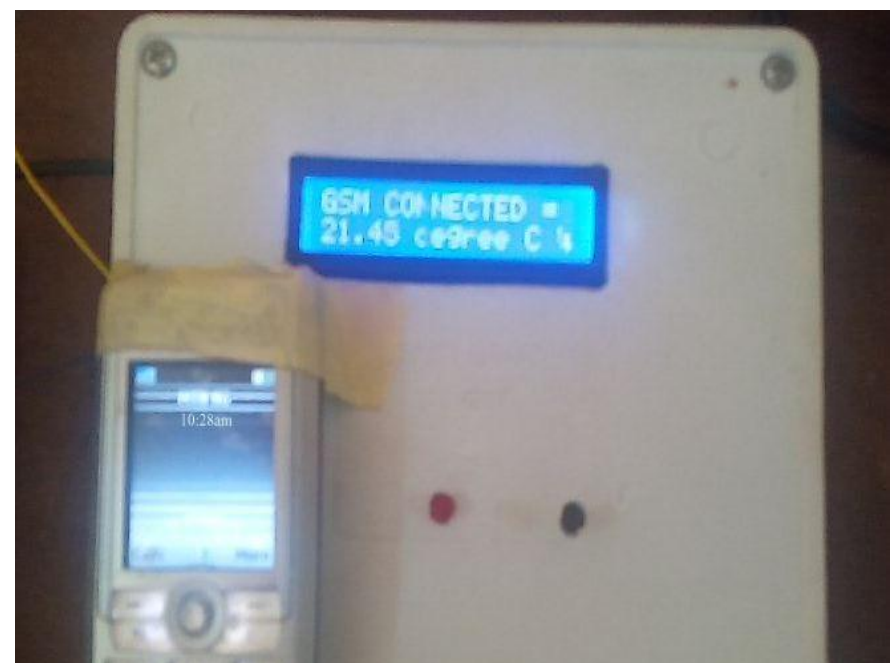

Plate1. Temperature read in the Morning

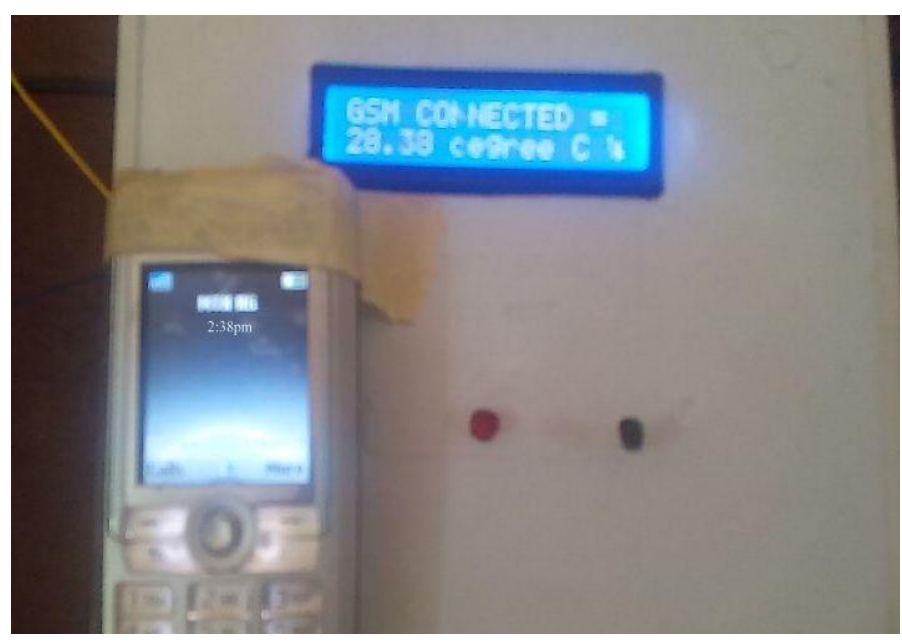

Plate2. Temperature read in the Afternoon

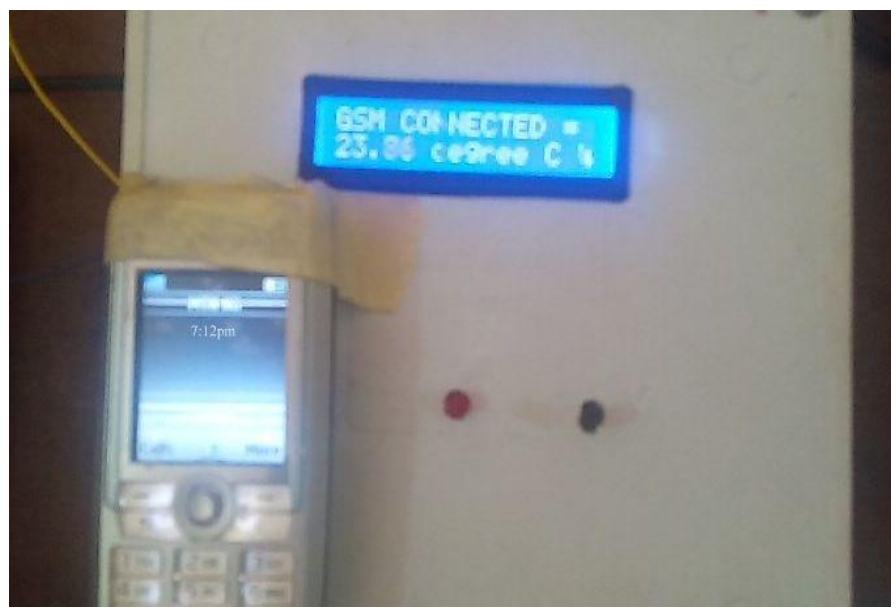

Plate3. Temperature read at Night 
Different Stages of the implementation are shown below: Plate 4 shows the environmental temperature. Plate 5 shows changes in temperature condition. Plate 6 shows the Labeled diagram of an Environmental monitoring system.

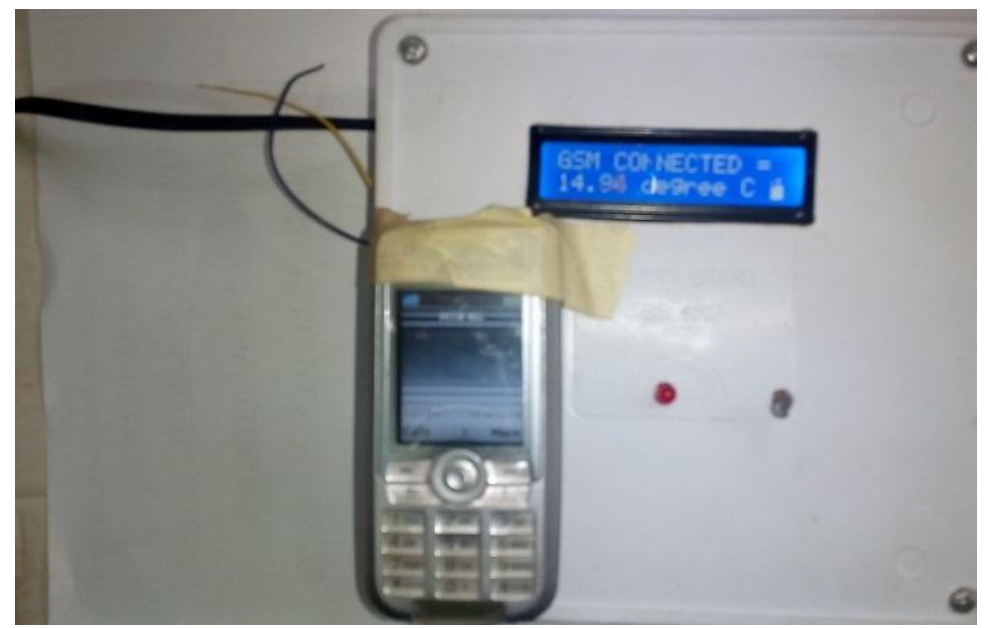

Plate4. Environment Temperature

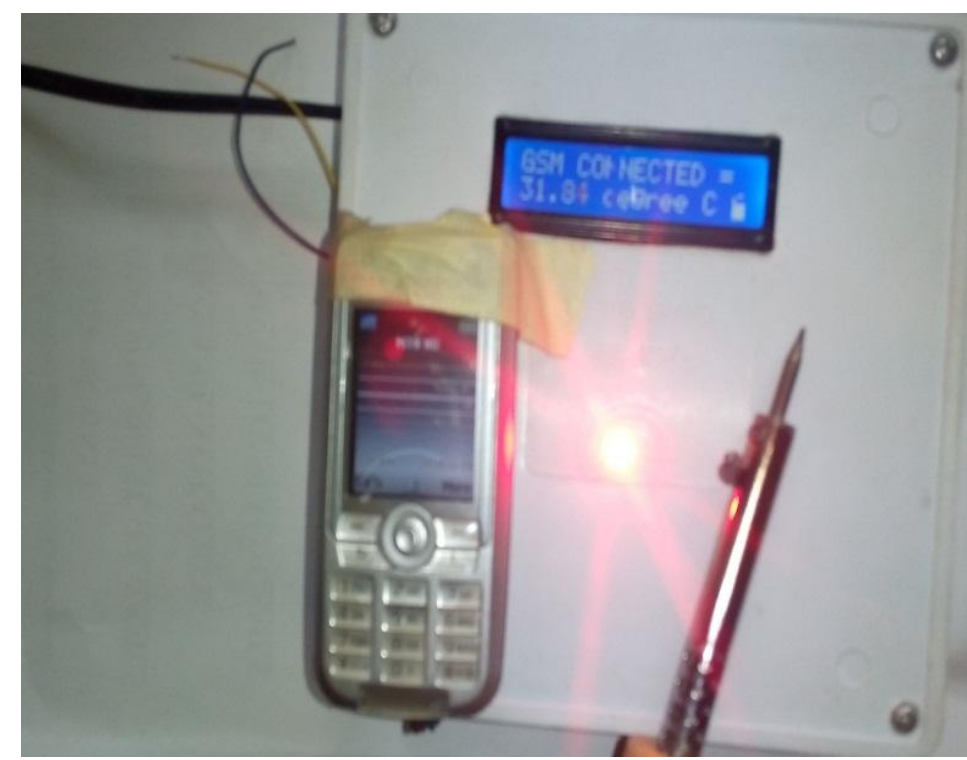

Plate5. Change in the temperature condition.

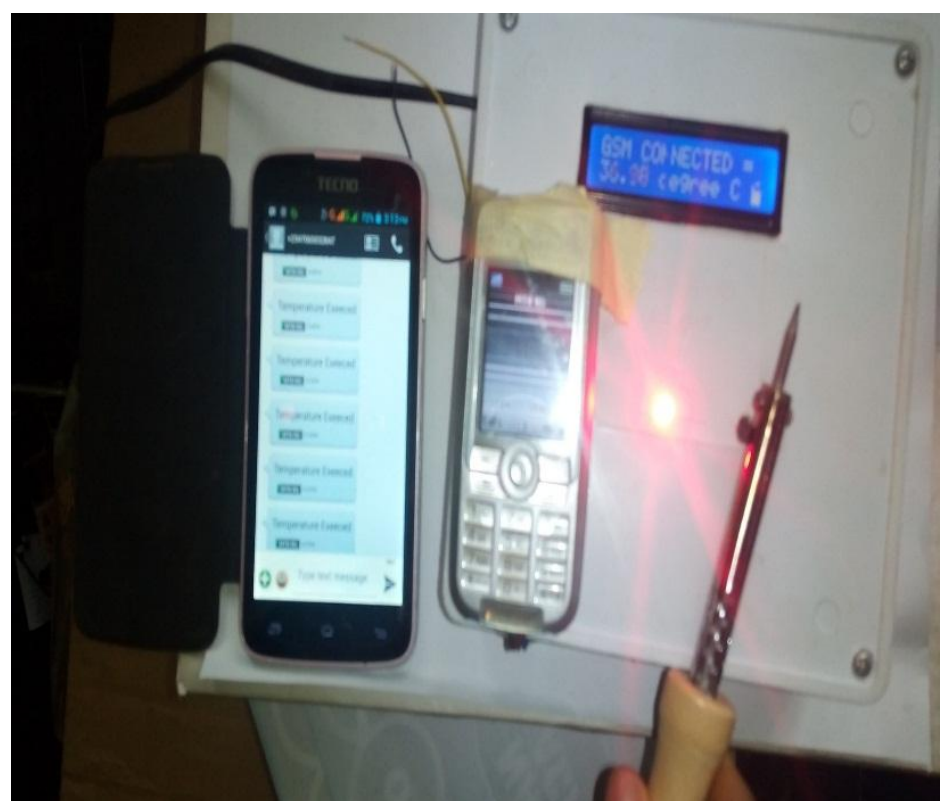

PI Plate6. Diagram of the Environmental Monitoring System 
Oke A.O. et al.

\section{CONCLUSION}

The construction of temperature sensor to monitor change in temperature was carried out considering its economic importance, availability of component to be used, efficiency, compatibility, portability and durability. The main objective of this work was achieved by designing and implementing an Environmental Monitoring system incorporated with an alert sub-system and a global system for mobile communication. Testing of the component to ensure it conforms to the requirement was followed by the construction on the circuit board. The circuit was assembled, tested and put to use. The EMS case was drilled for ventilation. Conclusively, it can be said that a fast, reliable and efficient system environmental monitoring system has been developed. It automatically monitors the environments temperature, and gives an audio signal when it exceeds the desired temperature and also sends an SMS to the person in charge.

\section{REFERENCES}

[1] Anies, Aprilson P., et.al. "A Global System for Mobile Communications Interface via Smoke and Fire Data Acquisition System" School Year 2008-2009

[2] G. Ramani, L. Nozal, A. Blazquez, and J. Arias, "Remote control of Sensors and Actuators by GSM", IEEE 2002, 28th Annual Conference of The Industrial Electronics Society IECON 02, vol. 3, 5-8 Nov. 2002, pp.2306 2310.

[3] S.Pandikumar, S.P.Kabilan and S.Ambethkar. 'Architecture of GSM based WSN for Greenhouse Monitoring System in Ambient Intelligence Environment'. International Journal of Computer Applications (0975-8887), Volume 63- No.6, February 2013.

[4] A.J Tseng, B. H. Sudantha, N. Kularatna 2006. "A Model for GSM Based Intelligence PC Monitoring System", International Journal of Advanced Computer Science and Technology, Volume 2, Number 2 (2012), pp. 85-88.

[5] Cao K., Butler, Z., Corke, P., Peterson, R., Rus, D., 2006. Virtual fences for controlling cows. In: Proceedings of the 2006 IEEE International Conference on Robotics and Automation, New Orleans, LA, USA, April 26-May 1, pp. 4429-4436.

[6] Li W, Z. Wei, Y.L. Zhou. "Sending and receiving short message by TC35i and person computer". Modern Electronics Technique. 30(15), 188-190 (2011).

[7] Palafox L, Islam, N.S. Wasi-ur-Rahman, M. An intelligent SMS-based remote Water Metering System. 12th International Conference on Computers and Information Technology, 2011, 21-23 Dec. 2011, Dhaka, Bangladesh.

[8] Usama Abdullah, Ayesha Ali (2014). GSM Based Water level and Temperature Monitoring System. International Journal of Recent Development in Engineering and Technology. Website: www.ijrdet.com(ISSN 2347-6435(Online) Volume 3, Issue 2, August 2014) 


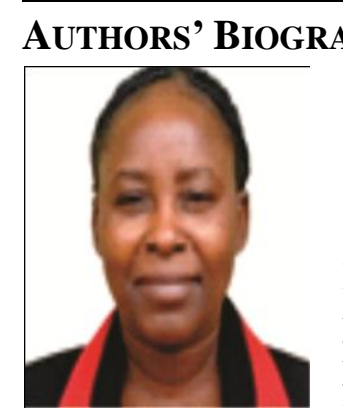

Oke Alice O., obtained B.Tech (Hons) Computer Engineering (1998), She obtained M.Tech Computer Science (2005) and bagged her PhD Computer Science (2012) in LadokeAkintola University of Technology (LAUTECH), Ogbomoso, Oyo State, Nigeria. She is currently a SENIOR LECTURER in the Department of Computer Science and Engineering of the same institution. A position she steadily rose to from Graduate Assistance. She has over 30 publications in reputable Journals and learned Conferences both locally and internationally. Her research interests are on signal and image engineering, hardware system design and implementation using wireless network of communication. Engr. Dr. Oke is a Registered Engineer with The Council for the Regulation of Engineering in Nigeria (COREN) and belongs to the following Professional bodies: Corporate member, Nigerian Society of Engineers (MNSE), full member of Computer Professional (Registration) Council of Nigeria, (MCPN) and the National Computer Society (NCS).

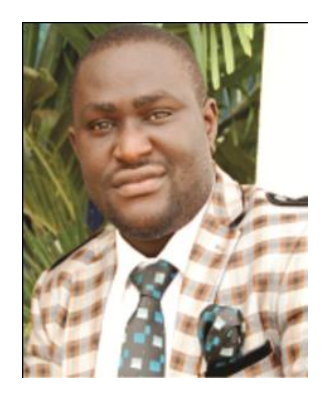

Awokola Jinmisayo A. holds a B.Tech (Hons), M.Tech and Ph.D in Computer Science from the department of Computer Science and Engineering, LadokeAkintola University of Technology, Ogbomoso, Nigeria. He has been teaching computer Science at both the undergraduate and postgraduate level for a few years. His current research interest is in software engineering, Cloud Computing as well as in the adoption of ICT related technologies for healthcare delivery.

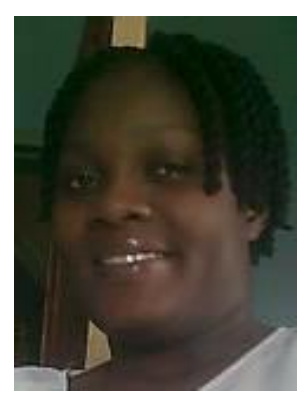

Amusan Elizabeth, A. currently lectures in the department of Computer Science and Engineering, LadokeAkintola University of Technology, Ogbomoso, Nigeria. She earned her B.Tech and M.Tech degrees in Computer Science in 2008 and 2011 respectively from the same institution. She is currently on her $\mathrm{Ph} . \mathrm{D}$. programme and a registered member of the Computer Professionals Reg. Council of Nigeria (CPN). Her current research interest is in software engineering, mobile ad-hoc networks and telemedicine technologies. 